INTERNATIONAL JOURNAL OF MULTIDisciplinARY RESEARCH AND ANALYSis

ISSN(print): 2643-9840, ISSN(online): 2643-9875

Volume 04 Issue 12 December 2021

DOI: 10.47191/ijmra/v4-i12-30, Impact Factor: 6.072

Page No.- 1990-1995

\title{
Comparative Study of the Anomalies of the Spermocytogram between two Automates: Sperm Class Analyzer (SCA) and Spermolyzer
}

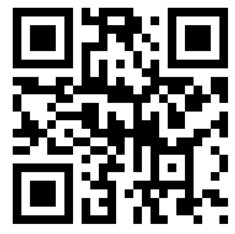

\author{
Rihab Bourchim ${ }^{1}$, Chaymaa Kabir-Idrissi ${ }^{1}$, Amina Benzaouiya ${ }^{1}$, Mustafa Zakaria ${ }^{3}$, Sabri yassir ${ }^{1}$, \\ Mohammed Zarqoui ${ }^{4}$, Achraf Zakaria ${ }^{1}$, Bouchra El Khalfi ${ }^{2}$, Abdelaziz Soukri ${ }^{2}$, Modou M. Mbaye ${ }^{1,2}$, \\ Noureddine Louanjli ${ }^{5}$ \\ ${ }^{1}$ Laboratory of Medical Analyses, Reproductive Biology, Labomac, Casablanca, Morocco \\ ${ }^{2}$ Laboratory of Physiopathology, Genetics Molecular and Biotechnology (PGMB), Faculty of Sciences Ain Chock, Research Center \\ Health and Biotechnology, University Hassan II of Casablanca, Morocco \\ ${ }^{3}$ Association for Scientific Research of the IRIFIV-AISRG Group, Consultant in IRIFIV Fertility Center, IVF laboratory, Casablanca, \\ Morocco
}

${ }^{4}$ GYN OBT IRIFIV Center, and IRIFIV-AISRG Scientific Research Group

${ }^{5}$ Labomac Clinical Analysis Laboratory, IRIFIV Fertility Center, AFC Fertility Center, Casablanca, Morocco

\begin{abstract}
Male infertility refers to the inability of a man to have children, after one year of regular unprotected sexual relations without contraceptive measures. The field of fertility has become a public health problem for some time, in this regard that it is now well established that the spermocytogram is a medical examination corresponding to the cytological and morphological analysis of spermatozoa, allowing the evaluation of male fertility. In our study, we compare the analysis of sperm with two automatons, the Sperm Class Analyzer (SCA) and the Spermolyzer, on 50 samples of sperm from voluntary men, with the aim of establishing the percentage of normal and abnormal spermatozoa and some specific anomalies, that is to say, the length and the identification of the shape. The results obtained indicate the accuracy and reliability of these automatons, showing more defined analyses with the use of the SCA, in contrast to the insignificant data rendered by the use of the Spermolyzer.
\end{abstract}

KEY WORD: SCA, Spermolyzer, male infertility, spermocytogram, spermatozoa.

\section{INTRODUCTION}

Infertility is defined as the absence of conception after at least 12 months of regular sexual intercourse without contraception [1], and for several years' infertility has become a worldwide scourge that affects about $15 \%$ of couples [2] of which $50 \%$ of cases are attributed to male factors [3].

Male infertility, or the inability of a man to reproduce, may be due to either quantitative sperm abnormalities (spermogram) and/or qualitative sperm abnormalities (spermocytogram) in connection with morphological abnormalities [4].

Spermocytogram is recognized as the morphological analysis of human spermatozoa including the evaluation of the percentage of morphologically normal spermatozoa and spermatozoa representing various morphological abnormalities [5].

Normal ejaculate contains spermatozoa with significant abnormalities in the size and shape of the head, acrosome, midpiece and flagellum as well as spermatozoa considered normal [6], and for this purpose, two automated systems were used to determine the percentage of normal and abnormal spermatozoa, as well as the percentages of all types of abnormal spermatozoa

The SCA Sperm Class Analyzer, which uses the CASA (Computer-Assisted Sperm Analysis) system, and which allows a repetitive and accurate automatic analysis of a sperm sample, and the Spermolyzer which is also a computer-assisted sperm analysis system that uses modern advanced computerized image processing techniques and is based on the WHO 5th criterion sperm testing standards (2010).

It is within this framework that our study is based on the correlation between the two spermiology automatons, the Sperm Class Analyzer and the Spermolyzer in order to .... 
Comparative Study of the Anomalies of the Spermocytogram between two Automates: Sperm Class Analyzer (SCA) and Spermolyzer

\section{MATERIALS AND METHODS}

\subsection{Semen sample collection}

The samples used in all the experiments of this work were collected at the Medical Analysis Laboratory, Reproductive Biology, Labomac, Casablanca, Morocco. This study was performed on 50 samples from male volunteers. Informed consent was obtained from all included patients before using their semen in this study. Samples were collected by masturbation after 3 to 4 days of abstinence in sterile, labeled containers.

Samples were stored and liquefied at $37^{\circ} \mathrm{C}$ until examination. We checked every 10 minutes until liquefaction was complete.

\subsection{Semen processing}

After liquefaction was complete, we performed macroscopic examination based on the criteria of volume, $\mathrm{pH}$, viscosity, appearance, and odor, followed by routine microscopic analysis according to World Health Organization (WHO-2010) standards and guidelines.

For the pretreatment of the samples, we used the protocol of morphological analysis of spermatozoa (Spermocytogram) which consists of centrifugation of $0.5 \mathrm{ml}$ of sperm plus $1 \mathrm{ml}$ of enrichment liquid (Earl) in the normal centrifuge at $1000 \mathrm{rpm}$ for $5 \mathrm{minutes}$ and then make a smear with the pellet, let it dry at room temperature and then perform the Shorr-hematoxillin staining. Thus, the smear was fixed with ethanol for 30 seconds, then the slide was covered with hematoxylin, washed after $5 \mathrm{~min}$, and covered again with Shorr reagent and washed after $3 \mathrm{~min}$. Finally, the slide was allowed to dry before being observed by the two automats, the Sperm Class Analyzer (SCA) and the Spermolyzer (figure 1).

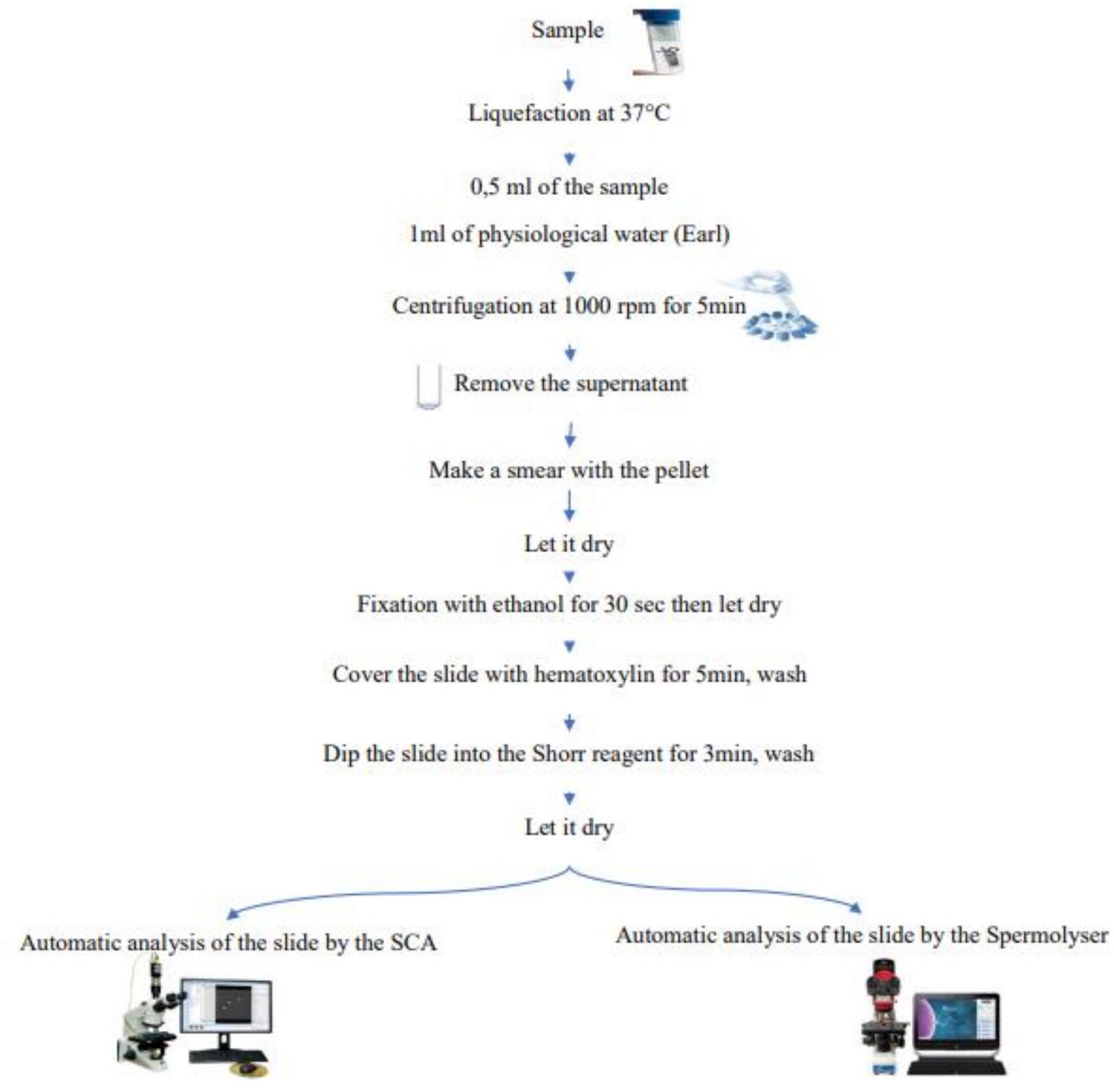

Figure 1: Sample processing treatment methodology 
Comparative Study of the Anomalies of the Spermocytogram between two Automates: Sperm Class Analyzer (SCA) and Spermolyzer

\subsection{Analysis on the CASA system}

A drop of immercient oil was placed on the stained smear and the slide was first mounted on the Sperm Class Analyser (SCA) microscope and observed in focus at x100 magnification. Secondly, the slide was mounted on the Spermolyzer microscope and observed at $x 100$ magnification. Morphology analysis of the 50 human sperm samples was done on both machines. The correlation between the two CASA systems was subsequently examined using the results obtained.

\subsection{Statistical analysis}

The data obtained in this experiment were statistically studied. The results of the correlation of the analysis of sperm morphology between the two CASA systems were performed by Student's t-test. All the graphs represented in this study were made with the software: GraphPadPrism.

\section{RESULTS}

\subsection{Normal spermatozoa}

The results of the percentage of normal spermatozoa showed a very large difference between the samples analyzed by the SCA compared to the Spermolyzer $(\mathrm{P}<0.05)$ (figure 2$)$.

\subsection{Abnormal Sperm}

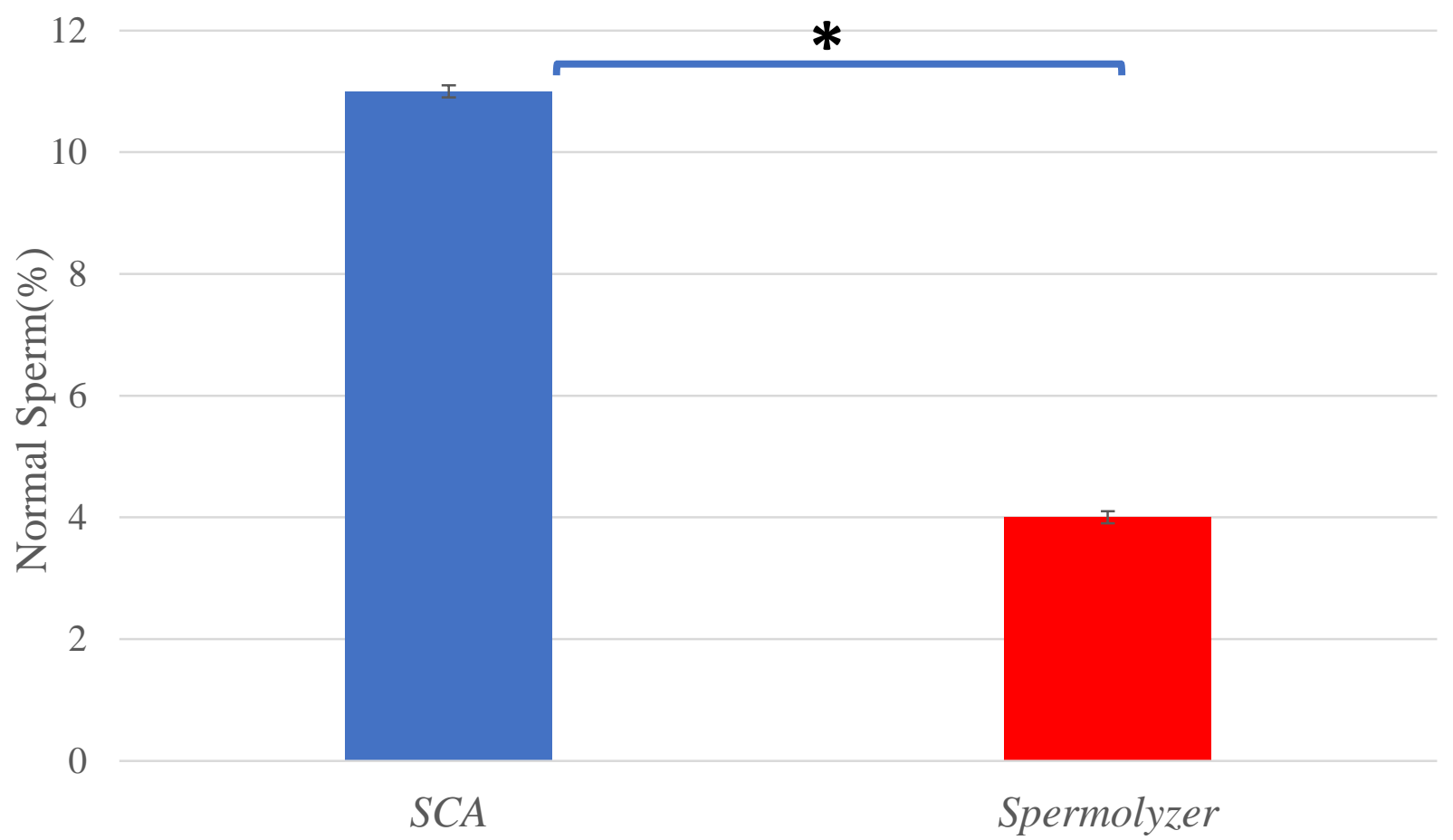

Figure 2: 1 Differences in manual and automatic SCA values of normal sperm

The results of the percentage of abnormal spermatozoa also showed a large difference between the samples analyzed by the SCA compared to the Spermolyzer $(\mathrm{P}<0.05)$ (figure 3$)$. 
Comparative Study of the Anomalies of the Spermocytogram between two Automates: Sperm Class Analyzer (SCA) and Spermolyzer

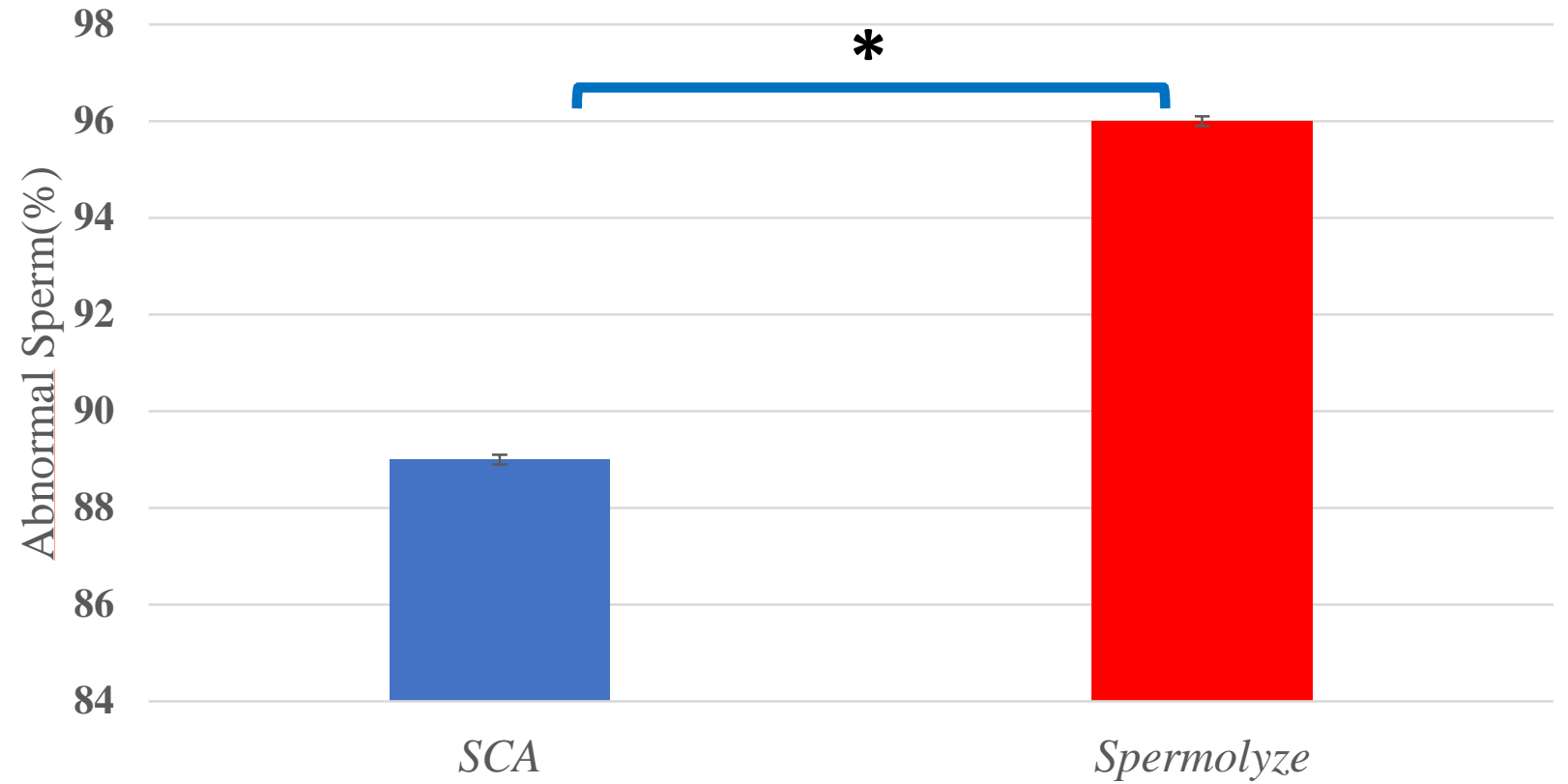

Figure 3 :Differences in manual and automatic SCA values of spermatozoa

\subsection{Spermatozoa with flagellar abnormalities}

The results of the percentage of spermatozoa representing flagellar abnormalities also showed a great difference between the samples analyzed by the SCA compared to the Spermolyzer ( $(\mathrm{P}<0.01)$ (figure 4$)$.

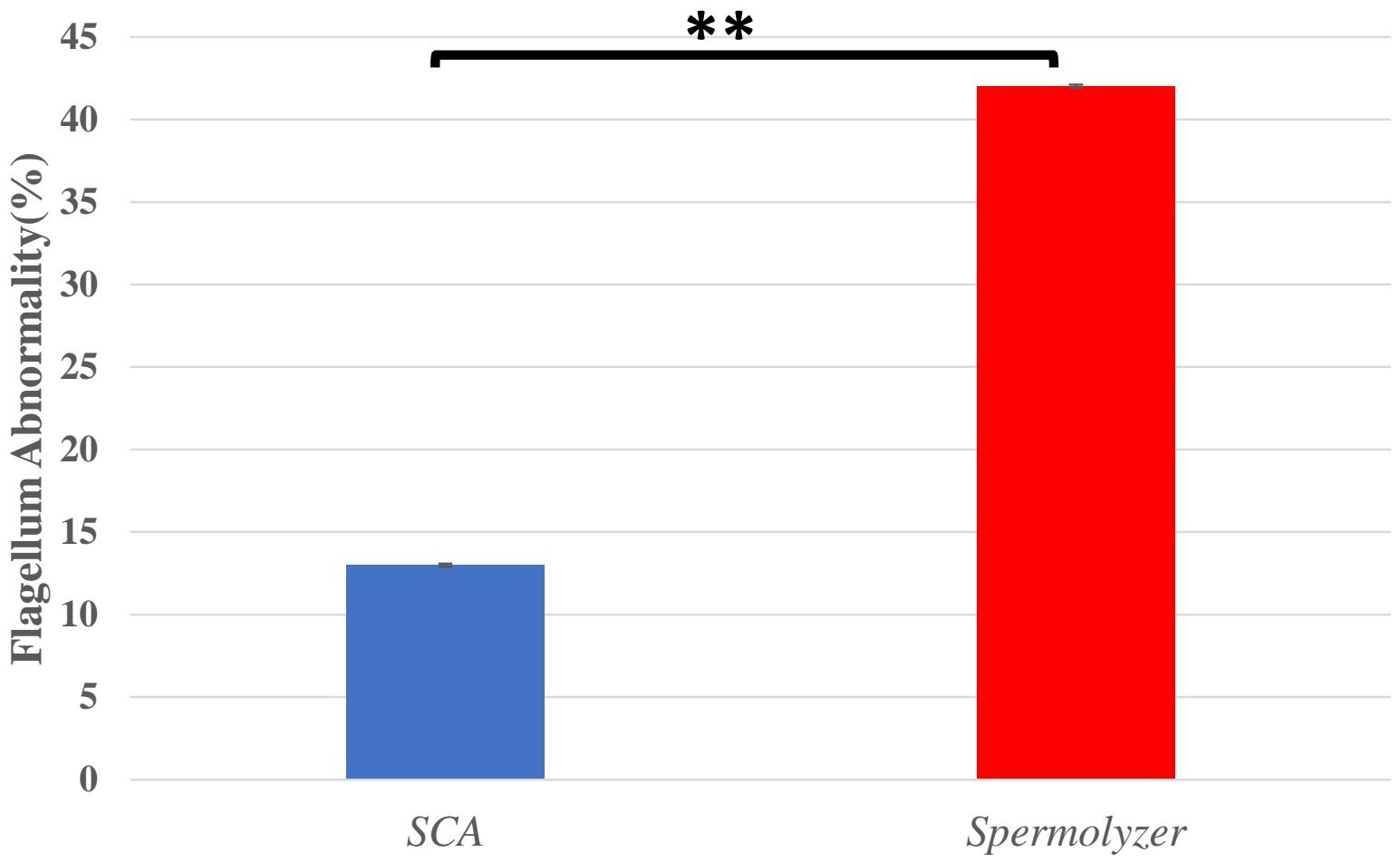

Figure 4 :Differences in manual and automatic SCA values of sperm flagellum anomalies

\subsection{Spermatozoa with cytoplasmic remains}

The results of the percentage of spermatozoa with cytoplasmic remains showed a minimal difference between the samples analyzed by the SCA compared to the Spermolyzer Spermolyzer $(P<0.05)$ (figure 5$)$. 
Comparative Study of the Anomalies of the Spermocytogram between two Automates: Sperm Class Analyzer (SCA) and Spermolyzer

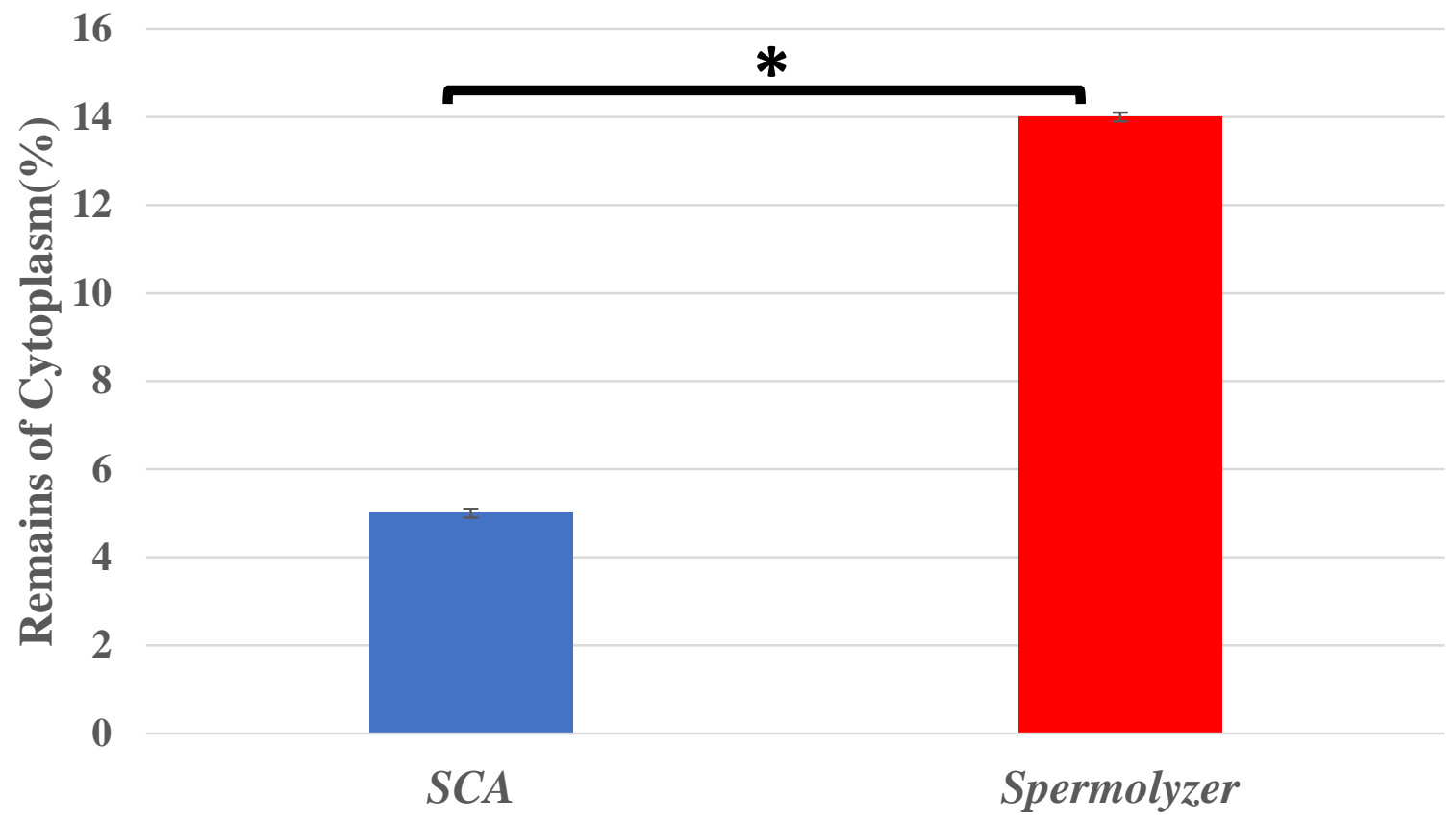

Figure 5: Differences in manual and automatic SCA values of cytoplasmic remains

\section{DISCUSSION}

The objective of our work is to make a comparative study of the results of the morphological evaluation of 50 semen samples between the two automatons Sperm Class Analyzer (SCA), and Spermolyzer in order to find a probable correlation between these two methods. The results of the percentage of normal spermatozoa showed a very large difference between the samples analyzed by the SCA compared to the Spermolyzer $(P<0.05)$ (figure 2). The results of the percentage of abnormal spermatozoa also showed a large difference between the samples analyzed by the SCA compared to the Spermolyzer $(P<0.05)$ (figure 3$)$. Concerning the analysis of flagella anomalies, there was also a large difference between the samples analyzed by the SCA compared to the Spermolyzer ( $\mathrm{P}<0.05$ ) (figure 4). Finally, the results of the percentage of spermatozoa with cytoplasmic residue showed a minimal difference between the samples analyzed by the SCA compared to the Spermolyzer $(P<0.01)$ (figure 5). Regarding the results of the head and midpiece anomalies, which showed clear and accurate percentages for the samples analyzed by the SCA, unlike the Spermolyzer system which is still not able to give results encompassing the final percentages of both types of anomalies mentioned above.

In accordance with the work of [7], who conducted a prospective study comparing spermocytogram analysis results between the two automata. For sperm morphology, both automated systems demonstrated high specificity (Sp) and negative predictive values (NPV), despite significantly different medians (CASA: $83.7 \%$ for Sp and 95.2\% for NPV, SQA-V: 97.9\% for Sp and 92.5\%). The highest accuracy (lowest $95 \%$ confidence interval for duplicate tests) for all semen variables was found in the SQA-V GOLD.

The results of our study regarding morphology, for all sperm abnormalities revealed a significant difference between the Sperm Class Analyzer (SCA) technique, and Spermolyzer. All this led us to say that our results are in perfect agreement with those of the study conducted by.

Conclusion

According to the research done by several analyzers, the results performed automatically are the most reliable, giving better correlations between the two CASA systems SCA and Spermolyzer. Our study highlights the comparison between these two automatons giving an automated analysis of sperm morphology called spermocytogram that allows a measurement of multiple morphometric parameters of spermatozoa. All this leads us to conclude that the Spermolyzer technique shows incomplete results, i.e. a lack of results of abnormalities of the head and the intermediate piece. In contrast to the SCA, which gives us significant and accomplished analyses.

\section{REFERENCES}

1) AUGUSTE, Yasmina. Etude génétique et fonctionnelle de l'infertilité masculine à propos de cas familiaux d'oligozoospermie et d'asthénozoospermie extrêmes. 2018. Thèse de doctorat. Aix-Marseille. 

and Spermolyzer

2) WALLERAND, Hervé, BERNARDINI, Stéphane, CHABANNES, Eric, et al. Infertilité masculine de cause génétique et biologie moléculaire. Progrès en urologie, 2003, vol. 13, p. 560-563.

3) SCHLOSSER, J., NAKIB, I., CARRÉ-PIGEON, F., et al. Infertilité masculine : définition et physiopathologie. In : Annales d'urologie. Elsevier Masson, 2007. p. 127-133.

4) BONDE, Jens Peter, GIWERCMAN, Aleksander, et ERNST, Erik. Identifying environmental risk to male reproductive function by occupational sperm studies: logistics and design options. Occupational and environmental medicine, 1996, vol. 53, no 8, p. 511-519.

5) AUGER, J., EUSTACHE, F., et DAVID, G. Standardisation de la classification morphologique des spermatozoïdes humains selon la méthode de David modifiée. Andrologie, 2000, vol. 10, no 4, p. 358-373.

6) SAÏDI, Rim, GRUEL, Elodie, ROSET-BLESSMAN, Julie, et al. Evaluation morphologique des spermatozoïdes. Andrologie, 2008, vol. 18, no 2, p. 158-167.

7) LAMMERS, J., SPLINGART, C., BARRIÈRE, P., et al. Double-blind prospective study comparing two automated sperm analyzers versus manual semen assessment. Journal of assisted reproduction and genetics, 2014, vol. 31, no 1, p. 35-43 